\title{
A Musculoskeletal Human Gait Model Using the Bond Graph Technique
}

\author{
R. Hernani ${ }^{1}$, G. Romero ${ }^{2}$, and M.L. Martinez ${ }^{2}$ \\ ${ }^{1}$ Departamento de Ingeniería Mecánico-Eléctirca, Universidad de Piura, Lima. Peru \\ ${ }^{2}$ Departamento de Ingenieria Mecátnica y Fabricación, Universidad Politécnica de Madrid. Madrid, Spain
}

\begin{abstract}
Today, the modeling and simulation of the human gait musculoskeletal system is well-developed using various techniques such as the vectorial mechanics and Lagrange's Equations, the Kane's method, etc. This paper proposes the use of the Bond Graph technique as an alternative method, developing primarily the muscle models needed to simulate, analyze and correct, in case of existing pathologies, the human gait motion. Two of the largest potentialities of the Bond Graph technique are their modular and multi-domain capacities. These capabilities facilitate the modification of the muscle parameters giving flexibility to the models and allowing a dynamic analysis of the gait. For each muscle, it has been used the Hill-type model that, with its schematic representation of the muscular functions such as the mechanical force generation, serial elastic, passive elastic, and damping elements, speeds up its implementation through the use of the Bond Graph technique.
\end{abstract}

Keywords- Movement Control, Bond Graph.

\section{INTRODUCTION}

This paper proposes a method to generate human gait motion based on the anatomy and physiology of the human body using the Bond Graph technique. The elements used in it, called "doors", focus on the transfer of energy through "flows" and "efforts" (for example, velocities and forces in the field of linear mechanics). The "graphs" combined suitably permit the incorporation of such effects as mechanical, electrical, thermodynamic, elastic, etc. into the dynamic system.

The data published by Delp [1] and Delp et al. [2] have been used in this paper. This data includes the attachment site of 43 muscles on each leg, physiological parameters such as the length of tendons, range of joints angles, etc. In Delp's research, several basic properties, such as the passive joint torque and the maximum isometric joint torque were calculated and compared with biomechanically measured data to evaluate the validity of the model. Other necessary data, such as the weight and the inertia of the body segments, were obtained from Yamaguchi and Zajac [3].

The developed model in this paper takes in account a 7 link segment model in the sagittal plane. Six of the segments represent the feet, shanks and thighs. The remaining segment is for the upper body -head, arms and trunk (HAT) - rigidly joined to the rest through the pelvis.
The model using this Bond Graph technique has been designed and simulated using the software Bondin $\odot$ Romero et al. [4].

\section{MusCLE MODEL}

A variety of mechanical models of muscle have evolved to describe and predict tension, based on some input stimulation. Crowe [5] and Gottlieb and Agarwal [6] proposed a contractile component in conjunction with a linear series and parallel elastic component plus linear viscous damper. Glantz proposed nonlinear elastic components plus a linear viscous component. Winter [7] has used a mass and a linear spring and damper system to simulate the second-order critically damped twitch. In 1949 Hill [8] proposed such a lumped-element model for the muscle. Hill's model is still in use today, and it remains the most popular form of lumped-element model for the muscle.

\section{A. The Hill-Type Model}

There are actually two canonical forms of Hill's model, but these forms are mathematically equivalent under suitable change of variables, McMahon [9]. The canonical form used here is the easier one to apply to a muscle when it is regarded as composed of multiple motor units acting in parallel with one another.

Figure 1 illustrates the Hill model. There are fout basic elements in it: 1) contractile element, $C$; 2) damping element, $B$; 3 ) series elastic component, $K_{S E}$; and 4 ) parallel elastic component, $K_{P E C}$.

Contractlle element: The contractile element $C$ is the "active" element in an extrafusal motor unit. It corresponds to the role played by voltage in an electronic circuit. $C$ responds to motoneuron inputs by contracting. Thus, the tension $T_{C}$ that it produces always acts to try to shorten the muscle. $C$ is incapable to produce an extension force.

The elastic elements: A muscle when passively stretched exhibits an elastic restoring force that tends to return the muscle to its original length. In part this force is due to stretching the connective tissue that surrounds the muscle fibers. In part it may be due to stretching the tendons which terminate muscle tissue and attach it to the bone. There is a reason to believe that the muscle fibers themselves are at 
least partly elastic. It is this elastic restoring force that is represented by the elastic elements (springs) in the Hill model. It is not completely correct to assign these elements to any particular physical source, but we may regard the $K_{P E C}$ as being mostly due to the connective tissues and the $K_{S E C}$ as being primarily dominated by tendon fibers terminating specific motor units. We should note that $K_{P E C}$ and $K_{S E C}$ are functions of lengths and therefore are non-linear springs.

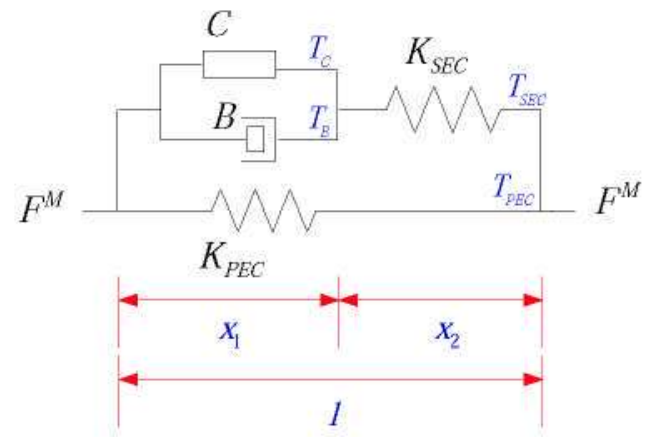

Fig. 1 Hill model

The damper element: It is an empirical factor that muscle tension during contraction and the speed of the contraction are coupled to each other. Hill found that the relation between them follows a characteristic hyperbolic equation, now known as Hill's equation. Such elastic elements, the damper coefficient $B$ is a function of the contraction speed, therefore is a nonlinear damper.

Wells [10] writes a complete set of equations describing each element.

\section{B. The Bond Graph Muscle Model}

The Hill muscle model showed in Figure 1 is represented with the Bond Graph technique using 1-Junctions for common velocity points, 0 -Junctions for common force points, an effort source $S_{e}$ for the contractile tension, a resistor element $R$ for the mechanical damper, and two capacitor elements $K$ for springs.

The full Bond Graph muscle model is shown in Figure 2. The linear force $\mathrm{F}^{\mathrm{M}}$ produced by a muscle can be transformed to an applied torque at a body joint through a modulated transformer element $M T F$ whose modulus $r$ reflects the moment arm from a muscle's attachment point center of rotation. This modulus $r$ is a function of the joint angel $\theta$ and depends on the physiological data of each muscle.

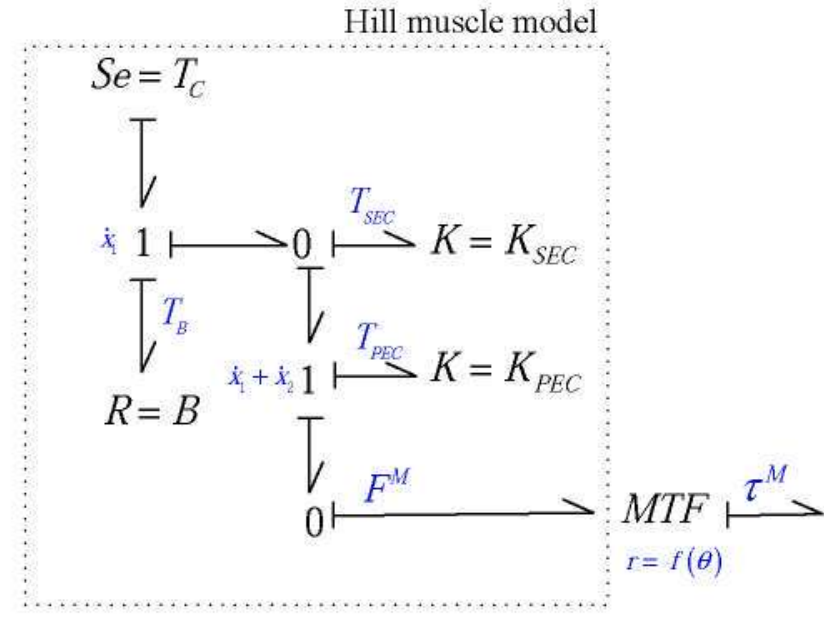

Fig. 2 Full Bond Graph muscle model

\section{Calculation of Muscle Force}

The method used to calculate the muscle force during de motion is explained in this section. First, joint torque is calculated with inverse dynamics from the motion. Joint torque is then decomposed to muscle force.

The force and torque at each joint can be calculated with the generalized coordinate parameters of the body. For this operation, called inverse dynamics, we have used a Bond Graph 7-segment model proposed by Hernani et al [11].

The torque $\tau_{i}$ made at joint $i$, is generated by the muscles crossing the joint:

$$
\tau_{i}=\sum_{j} r_{j} \times f_{j}
$$

where $r_{j}$ and $f_{j}$ are the moment arm and the force exerted by muscle $j$, respectively. However, since the number of muscles crossing joint $i$ is always greater than the DOF of the joint, solving $f_{j}$ in (1) is a redundant problem. We apply an optimization method to determine the muscle force (Crowninshield and Brand [12]; Komura et al. [13]). A criterion:

$$
u=\sum_{i=1}^{i_{m}}\left(\frac{f_{i}^{t}}{A_{j}}\right)^{2}
$$

where $f_{i}^{t}=\left|f_{j}\right|, A_{i}$ is the PCSA of muscle $i$, and $n_{m}$ is the number of muscles to be optimized. We use (1) as equality constraints and (2) as inequality constraints, so that $u$ can be minimized by quadratic programming. The muscle force $f$ at this moment is obtained at the same time. However, we do not use the upper limit $f \leq f^{\text {max }}$ here. This constraint will be used in future research to obtain physiologically feasible motion (Komura et al. [14]). 


\section{FULL BOND GRAPH MUSCULOSKELETAL MODEL}

One of the most important features of the Bond Graph technique is its modular capacity. The torque applied to the joint is the sum of the $n_{m}$ muscles crossing it. Using Bond Graph, a 1-Junction performs this function. Figure 4 shows $n_{m}$ muscles applying a full torque $\tau_{i}$. Each modular block BBmm represents the Bond Graph full muscle model shown in Figure 3.

The full 7-segment musculoskeletal model using Bond Graph technique is showed in Figure 4.

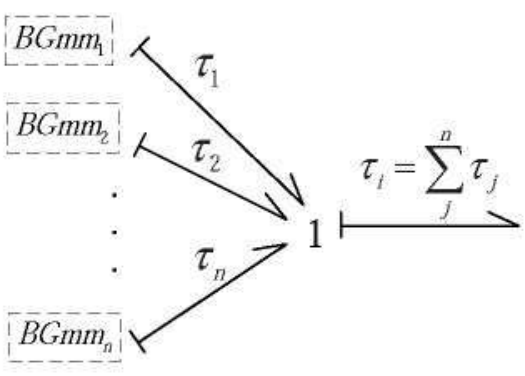

Fig. 3 Bond Graph for $n_{m}$ muscles crossing a joint

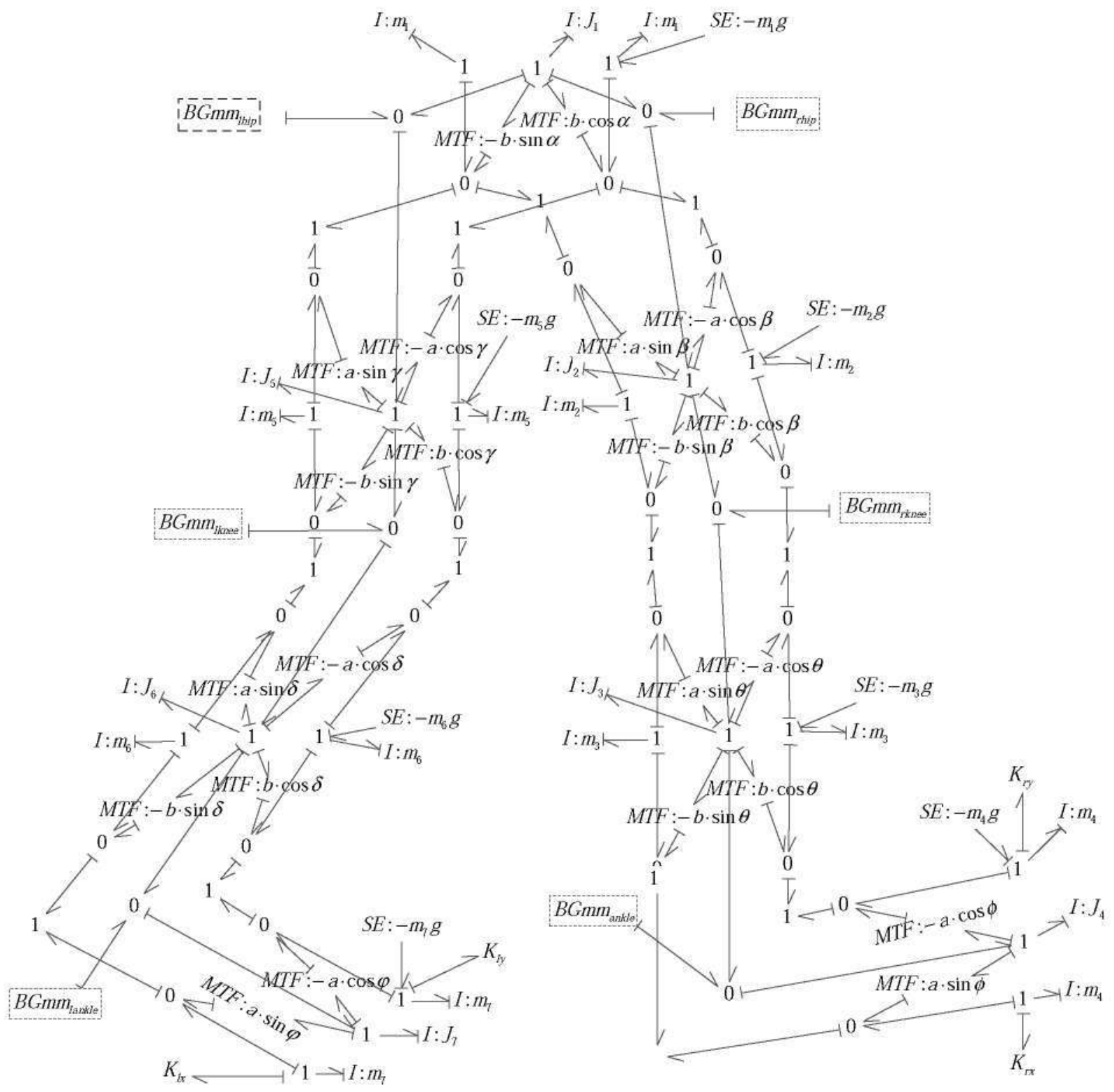

Fig. 4 Full Bond Graph musculoskeletal model 
This Bond Graph musculoskeletal model shows the full torque applied to each joint as modular blocks which represent the Bond Graph for the $n_{m}$ muscles crossing the joint (Figure 4). On both feet we have added springs that only work when each foot rests on the floor. These capacitor elements will enable us to calculate the ground reaction forces $G R F$ ( Figures 5 and 6).

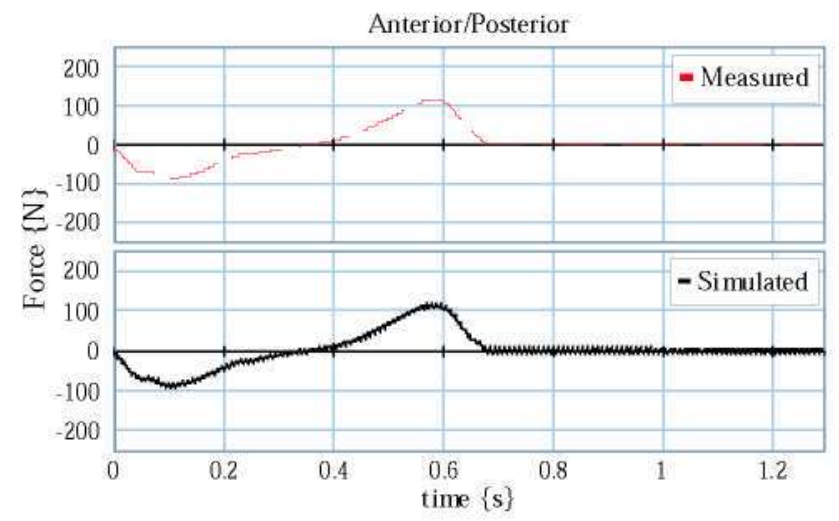

Fig. 5 Right leg Anterior/Posterior ground reaction force

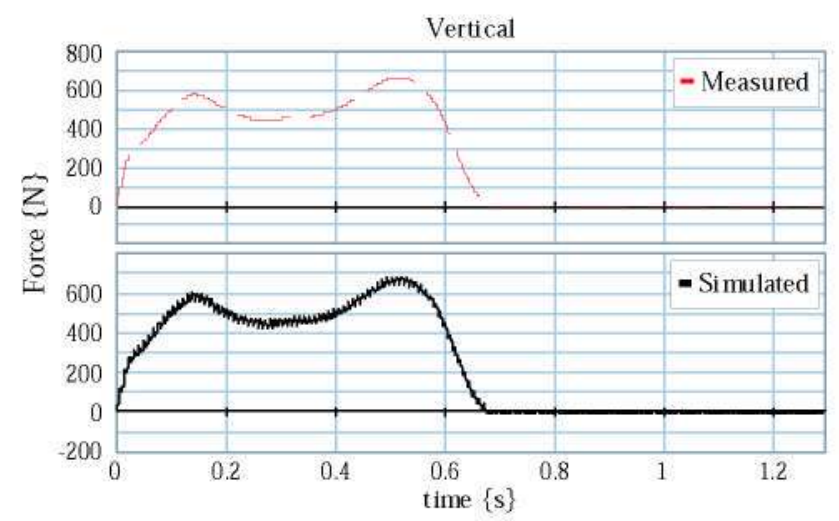

Fig. 6 Right leg Vertical ground reaction force

\section{Conclusions}

For the dynamic analysis of connected segment systems, mathematical models consisting of interconnected mass elements, springs, dampers, and actuators are often used. These types of mathematical models speed up the implementation of the Bond Graph technique.

The human gait musculoskeletal system is welldeveloped using various techniques such as Lagrangian dynamics or the Kane's method. However, the Bond Graph technique offers a new alternative to research the human gait musculoskeletal model. Bond Graph has the benefit of being easily integrated with similarly developed external controllers and implantable electromechanical devices. Also, the common Bond Graph language allows for the development and cataloguing of standard modules that can be easily integrated in new applications.

This paper shows the great potential that the technique of Bond Graph offers for modeling the human gait motion, basically by their modular and multi-domain capacities.

\section{REFERENCES}

1. Delp S (1990) Surgery simulation: a computer graphics system to analyze and design musculoskeletal reconstructions of the lower limb. $\mathrm{PhD}$ Thesis, The Department of Mechanical Engineering, Stanford University, Stanford, Calif.

2. Delp S, Loan P, Hoy M, Zajac FE, Fisher S, Rosen J (1990) An interactive graphics-based model of the lower extremity to study orthopaedic surgical procedures. IEEE Trans Biomed Eng 37:757-767

3. Yamaguchi DG, Zajac FE (1990) Restoring unassisted natural gait to paraplegics via functional neuromuscular stimulation: a computer simulation study. IEEE Trans Biomed Eng 37:886-902

4. Romero, G., Félez, J., Cabanellas, J.M., Maroto., J.; BONDIN: A new engineering simulation software for $\mathrm{ODE}$ and DAE systems with symbolic notation based in the Bond Graph technique, 8th WSEAS International Conference on Software Engineering, Parallel And Distributed Systems, Cambridge, United Kingdom, February 2009. pp. $90-97$

5. Crowe, A. (1970) A Mechanical Model of Muscle and its Application to the Intrafusal Fibres of Mammalian Muscle Spindle, J Biomech $3: 583,592$

6. Gottlieb, G. L. and G. C. Agarwal (1971) Dynamic Relationship between Isometric muscle Tension and the Electromyogram in Man, J Appl Physiol 30:343-351

7. Winter, D. A. (1976) Biomechanical Model Related EMG to Changing Isometric Tension, in Dig. $11^{\text {th }}$ Int. Conf. Med. Biol. Eng. pp. 362 363

8. Hill A. V. (1949) The abrupt transition from rest to activity in muscle, Proc. Roy. Soc. London B, vol. 136, issue 884, pp. 399-420

9. McMahon T.A.(1984) Muscles, Reflexes, and Locomotion, Princeton, NJ: Princeton University Press, pp. 23-25

10. R. Wells, (2003) Kinetics and Muscle Modeling of a Single Degree of Freedom Joint, Part I: Mechanics, at http://www2.cs.uidaho.edu/-tsoule/website_with_hierarchy/document s_page.html

11. R. Hernani, G. Romero, R. Jazmati, An alternative for human gait modelling using the Bond Graph technique, IFToMM Proc. Interdisciplinary Applications of Kinematics, Lima, Peru 2008, pp. 161-173

12. Crowninshield RD, Brand RA (1981) A physiologically based criterion of muscle force prediction in locomotion. J Biomech 14:793-800

13. Komura T, Shinagawa Y, Kunii TL (1997) Muscle-based feedforward controller of the human body. Comput Graph Forum 16:C165-C176

14. Komura T, Shinagawa Y, Kunii TL (2000). Creating and retargeting motion by the musculoskeletal human body model. The Visual Computer 16:254-270 